\title{
Solvent-free one-pot 1,3-dipolar cycloaddition reactions of dihydropyran derived nitrone
}

\author{
BHASKAR CHAKRABORTY*, PRAWIN KUMAR SHARMA, NEELAM RAI \\ and CHIRAN DEVI SHARMA \\ Organic Chemistry Laboratory, Sikkim Government College, Gangtok 737 102, India \\ e-mail: bhaskargtk@yahoo.com
}

MS received 4 May 2011; revised 19 October 2011; accepted 31 October 2011

\begin{abstract}
Microwave-induced 1,3-dipolar cycloaddition reactions of dihydropyran derived nitrone with various activated alkenes have been studied in situ and found to afford new isoxazolidine derivatives with moderate selectivity.
\end{abstract}

Keywords. Solid phase; cycloaddition reaction; isoxazolidines and amino alcohols.

\section{Introduction}

The synthetic utility of microwave irradiation in organic synthesis has increased considerably in recent years. ${ }^{1-5}$ This non-conventional energy source is able to reduce chemical reaction time and to increase yield and in some cases leads to better result than those obtained with conventional heating methods. Microwave reactions are quite often cleaner, faster, and higher-yielding than conventional ones. This methodology can be regarded as environmental friendly, mainly because solvent-free reactions are especially suited to microwave conditions. ${ }^{6,7}$ Microwave technology has been successfully used to perform difficult cycloadditions and to obtain temperature sensitive compounds. ${ }^{8-13}$ Particularly interesting part is 1,3-dipolar cycloaddition, which represents one of the most versatile tools for the construction of five-membered heterocycles. Owing to the labile nature of the $\mathrm{N}-\mathrm{O}$ bond under mild reducing conditions, isoxazolidines provide easy access to a variety of fascinating 1,3-difunctional amino alcohols. ${ }^{14}$ In continuation of our green methodological synthesis of spiro isoxazolidine, isoxazolidine, isoxazoline, aldehyde, ketone synthesis using $\alpha$-chloro and $\alpha$-amino nitrones in solid phase and in hydrated media, ${ }^{15-23}$ we report here microwave-assisted 1,3-dipolar cycloaddition reactions of dihydropyran derived nitrone leading to the green synthesis of new isoxazolidine derivatives with moderate selectivity and excellent yield (scheme 1, table 1).

*For correspondence
Literature survey reveals that this is quite a new approach of synthesis of nitrone from dihydropyran using microwave irradiations. ${ }^{24-26}$

Synthetic potential of the new isoxazolidine derivatives (2-6) are tremendous as they could be converted into acyclic chiral 1,3 difunctional amino alcohols (scheme 2, table 2) by the reductive cleavage of the $\mathrm{N}-\mathrm{O}$ bond by treatment with zinc powder in dil acetic acid under microwave irradiation. ${ }^{27-29}$

\section{Experimental}

\subsection{General procedures}

Melting points were determined in open capillary tubes and are uncorrected. ${ }^{1} \mathrm{H}$ NMR spectra were recorded with a Bruker Avance DRX-300 spectrometer (300 MHz, FT NMR) using TMS as internal standard. ${ }^{13} \mathrm{C}$ NMR spectra were recorded on the same instrument at $75 \mathrm{MHz}$. The coupling constants $(J)$ are given in Hz. IR spectra were obtained with a Perkin-Elmer RX 1-881 machine as $\mathrm{KBr}$ pellets for all the products. MS spectra were recorded with a Jeol SX-102 (FAB) instrument. Elemental analyses $(\mathrm{CHN})$ were performed with a Perkin-Elmer 2400 series CHN analyzer. TLC's were run on Fluka silica gel pre-coated TLC plates. All other reagents and solvents were purified after receiving from commercial suppliers. $N$-phenylhydroxylamine was prepared following standard methods available in literature. Microwave irradiations were carried out using domestic microwave oven KENSTER (19LKH, 19SSLM, $800 \mathrm{~W}-2450 \mathrm{MHz}$ ). 


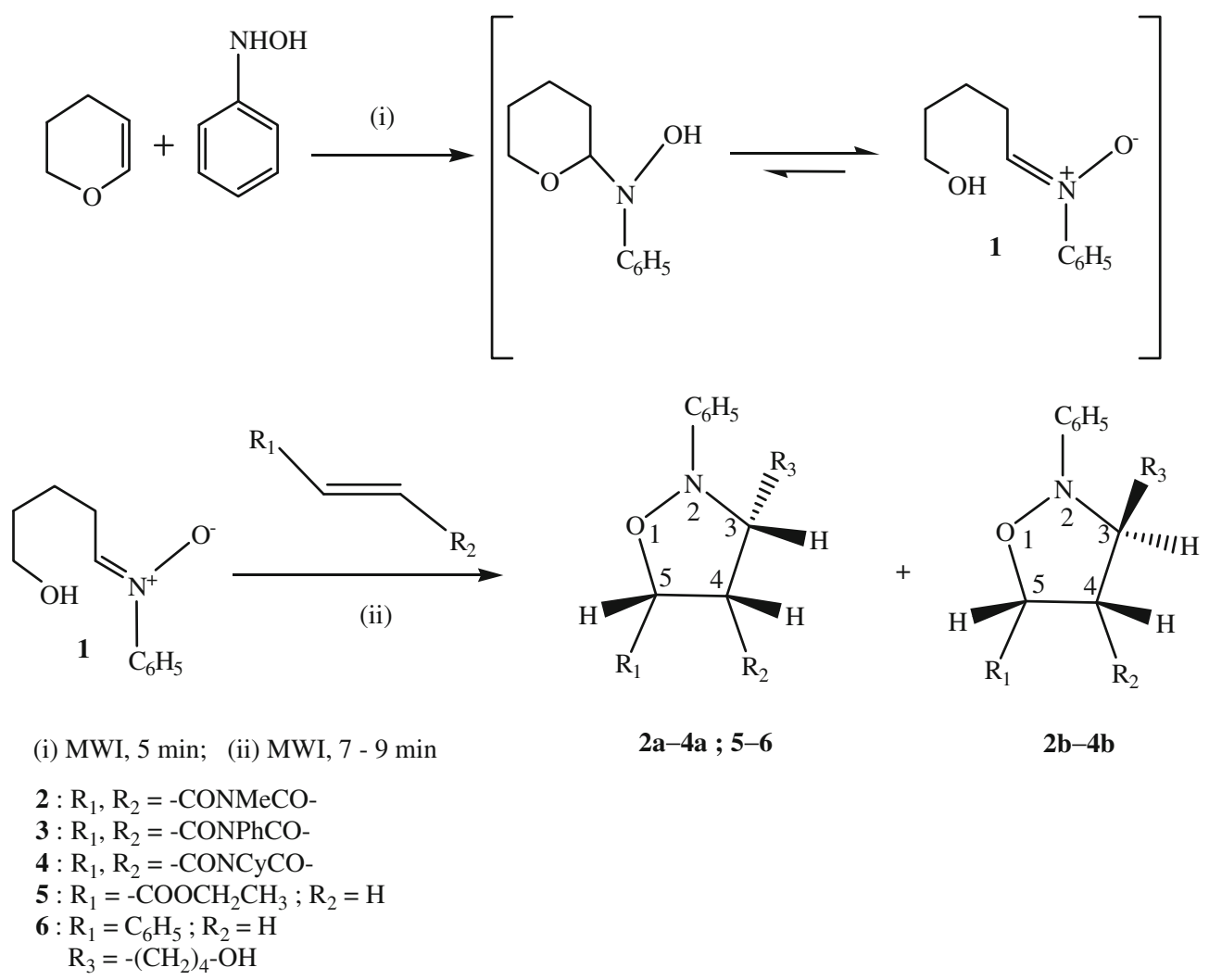

Scheme 1. Synthesis of novel isoxazolidine derivatives.

2.1a General procedure for cycloaddition reaction (conventional): $N$-phenylhydroxylamine $\quad(250 \mathrm{mg}$, $2.29 \mathrm{mmol}$ ) was added to a solution of 2,3-dihydro- $4 \mathrm{H}$ pyran $(192 \mathrm{mg}, 1$ equivalent) in dry benzene $(20 \mathrm{~mL})$ under $\mathrm{N}_{2}$ atmosphere and the reaction mixture was refluxed for $24 \mathrm{~h}$ while the progress of the reaction was monitored by TLC (hexane:ethyl acetate $=5: 1 ; \mathrm{R}_{f}=$
0.38). Dipolarophiles were added (1 equivalent) at this stage and the reaction mixture was further refluxed for 13-15 h. The solvent was evaporated off and the cycloadducts (2-6; table 1) were isolated by column chromatography using benzene-pet ether as eluent. But this methodology was discarded because of lengthy reaction process, poor yield and use of benzene as solvent.

Table 1. Physicochemical data of synthesized compounds $(\mathbf{2} \mathbf{a}-\mathbf{4 a} ; \mathbf{5}-\mathbf{6}$ and $\mathbf{2 b}-\mathbf{4 b})$.

\begin{tabular}{|c|c|c|c|c|c|c|}
\hline Entry & Nitrone & Dipolarophile $^{\mathrm{a}}$ & Time (min) & $\begin{array}{l}\text { Cycloadduct and m.p }\left({ }^{\circ} \mathrm{c}\right) \\
\mathbf{2 a}-\mathbf{4 a} \text { : } \text { cis; } \mathbf{2 b}-\mathbf{4 b} \text { : } \text { trans }\end{array}$ & $\begin{array}{l}\text { Cis/trans } \\
\text { ratio }(\%)\end{array}$ & Yield $^{\mathrm{b}}(\%)$ \\
\hline 1 & $\begin{array}{l}N \text {-phenyl-5- } \\
\text { hydroxy nitrone }\end{array}$ & $\begin{array}{l}N \text {-methyl } \\
\text { maleimide }\end{array}$ & $7(13 \mathrm{~h})$ & $\begin{array}{l}\text { 2a: White crystal, } 95 \\
\text { 2b: White crystal, } 82\end{array}$ & $\begin{array}{l}\mathbf{2 a}: 64 \\
\mathbf{2 b}: 32\end{array}$ & $96(54)$ \\
\hline 2 & $\begin{array}{l}N \text {-phenyl-5- } \\
\text { hydroxy nitrone }\end{array}$ & $\begin{array}{l}N \text {-phenyl } \\
\text { maleimide }\end{array}$ & $9(14 \mathrm{~h})$ & $\begin{array}{l}\text { 3a: Pale yellow solid, } 108 \\
\text { 3b: White solid, } 103\end{array}$ & $\begin{array}{l}\text { 3a: } 68 \\
\text { 3b: } 25\end{array}$ & $93(52)$ \\
\hline 3 & $\begin{array}{l}N \text {-phenyl-5- } \\
\text { hydroxy nitrone }\end{array}$ & $\begin{array}{l}N \text {-cyclohexyl } \\
\text { maleimide }\end{array}$ & $9(14 \mathrm{~h})$ & $\begin{array}{l}\text { 4a: Yellow crystal, } 80 \\
\text { 4b: Yellow crystal, } 88\end{array}$ & $\begin{array}{l}\mathbf{4 a}: 62 \\
\mathbf{4 b}: 31\end{array}$ & $93(47)$ \\
\hline 4 & $\begin{array}{l}N \text {-phenyl-5- } \\
\text { hydroxy nitrone }\end{array}$ & Ethyl acrylate & $9(15 \mathrm{~h})$ & 5: Colourless thick liquid & & $92(56)$ \\
\hline 5 & $\begin{array}{l}N \text {-phenyl-5- } \\
\text { hydroxy nitrone }\end{array}$ & Styrene & $9(15 \mathrm{~h})$ & 6: Colourless thick liquid & & $91(50)$ \\
\hline
\end{tabular}

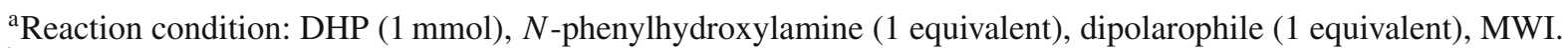

${ }^{\mathrm{b}}$ Isolated yields after purification.

Figures in parentheses indicate reactions performed in conventional method 


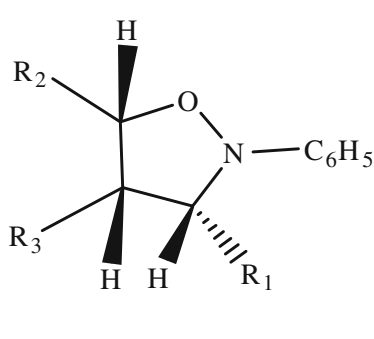

$2 a-4 a$

(i) MWI, 9 - $10 \mathrm{~min}$

$2: \mathrm{R}_{2}, \mathrm{R}_{3}=-\mathrm{CONMeCO}-$

$3: \mathrm{R}_{2}, \mathrm{R}_{3}=-\mathrm{CONPhCO}-$

$4: \mathrm{R}_{2}, \mathrm{R}_{3}=-\mathrm{CONCyCO}$ -

$\mathrm{R}_{1}=-\left(\mathrm{CH}_{2}\right)_{4}-\mathrm{OH}$

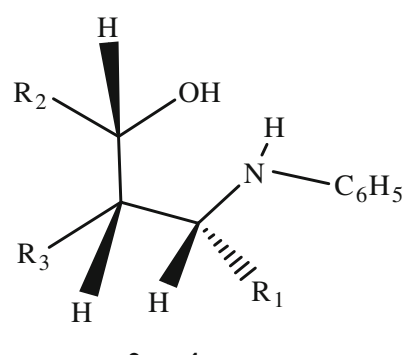

$2 c-4 c$

Scheme 2. Synthesis of 1,3 amino alcohols.

2.1b General procedure for cycloaddition for diastereomers (MWI): A mixture of $N$-phenylhydroxylamine (250 mg, $2.29 \mathrm{mmol})$, 2,3-dihydro-4H-pyran (192 mg, 1 equivalent) was taken in a $25 \mathrm{~mL}$ Erlenmeyer flask, mixed well and subjected to microwave irradiation at $250 \mathrm{~W}$ for $5 \mathrm{~min}$. The formation of nitrone was monitored by TLC (hexane: ethyl acetate $=5: 1 ; \mathrm{R}_{f}=0.38$ ). $\mathrm{N}$-methyl maleimide ( $254 \mathrm{mg}$, 1 equivalent) was added at this stage and the reaction mixture was further irradiated at $250 \mathrm{~W}$ for an appropriate time (table 1). After completion of the reaction, as indicated by TLC (hexane:ethyl acetate $=5: 1 ; \mathrm{R}_{f}=0.44,0.50$ ), the reaction mixture was cooled to room temperature (RT) and washed with diethyl ether $(3 \times 10 \mathrm{~mL})$. The combined ether extract was concentrated in vacuo and the resulting products were directly charged on silica gel column and eluted with a mixture of ethyl acetate:hexane to afford pure isoxazolidine derivatives $\mathbf{2 a}$ and $\mathbf{2 b}$ (entry 1 , table 1). This procedure was followed for other substrates listed in table 1.

2.1c Dihydro-3-(4-hydroxybutyl)-5-methyl-2-phenyl2H-pyrrolo[3,4-d]isoxazole-4,6(5H,6a-H)-dione (2a): White crystal. Yield 64\%; $\mathrm{R}_{f}=0.44$ (hexane-ethyl acetate, 5:1); IR (KBr): $v_{\max } 3640-3530$ (br), 2915 (m), 2832 (m), 1774 (s), 1680 (s), 1440 (m), 1380 (m), $772(\mathrm{~s}) \mathrm{cm}^{-1} ;{ }^{1} \mathrm{H} \mathrm{NMR}\left(\mathrm{CDCl}_{3}, 300 \mathrm{MHz}\right): \delta 6.88$ $6.73\left(\mathrm{~m}, 5 \mathrm{H}, \mathrm{C}_{6} \mathrm{H}_{5}\right), 4.83$ (br, s, $1 \mathrm{H}, \mathrm{OH}$, exchanged in $\left.\mathrm{D}_{2} \mathrm{O}\right), 4.64\left(\mathrm{~d}, 1 \mathrm{H}, J=6.30 \mathrm{~Hz}, \mathrm{C}_{5} \mathrm{H}\right), 4.23(\mathrm{dd}, 1 \mathrm{H}$, $\left.J=6.06,6.04 \mathrm{~Hz}, \mathrm{C}_{4} \mathrm{H}\right), 3.30\left(\mathrm{~s}, 3 \mathrm{H}, \mathrm{CH}_{3}\right.$ protons $)$, $2.78\left(\mathrm{dt}, 1 \mathrm{H}, J=6.40,6.54 \mathrm{~Hz}, \mathrm{C}_{3} \mathrm{H}\right), 2.36(\mathrm{dt} \sim \mathrm{m}$, $2 \mathrm{H}, \mathrm{CH}_{2}$ protons of $\left.-\mathrm{CH}_{2}-\left(\mathrm{CH}_{2}\right)_{3} \mathrm{OH}\right), 1.80-1.43(\mathrm{~m}$, $6 \mathrm{H}, \mathrm{CH}_{2}$ protons $) ;{ }^{13} \mathrm{C} \mathrm{NMR}\left(\mathrm{CDCl}_{3}\right): \delta 176.22,176.10$ (carbonyl carbons), 130.62, 130.16, 128.88, 128.15 (aromatic carbons), $87.15\left(\mathrm{C}_{5}\right), 76.42\left(\mathrm{C}_{3}\right), 66.23$ $\left(\mathrm{CH}_{2} \mathrm{OH}\right), 55.50\left(\mathrm{C}_{4}\right), 38.00\left(\mathrm{CH}_{3}\right), 25.76,22.31,20.27$ (3 $\mathrm{CH}_{2}$ carbons); FAB-MS: $m / z 304\left(\mathrm{M}^{+}\right), 289,231$, 227, 212, 154 (B.P), 77, 73; Anal. Found: C, 63.02; H, 6.35; N, 9.17. $\mathrm{C}_{16} \mathrm{H}_{20} \mathrm{O}_{4} \mathrm{~N}_{2}$ requires $\mathrm{C}, 63.13 ; \mathrm{H}, 6.61$; $\mathrm{N}, 9.21 \%$.

2.1d Dihydro-3-(4-hydroxybutyl)-5-methyl-2-phenyl2H-pyrrolo[3,4-d]isoxazole-4,6(5H,6a-H)-dione (2b): White crystal. Yield $32 \% ; \mathbf{R}_{f}=0.50$ (hexane-ethyl acetate, 5:1); IR (KBr): $v_{\max }$ 3645-3550 (br), 2910 (m), $2830(\mathrm{~m}), 1776(\mathrm{~s}), 1676(\mathrm{~s}), 1440(\mathrm{~m}), 1385(\mathrm{~m})$, $775(\mathrm{~s}) \mathrm{cm}^{-1} ;{ }^{1} \mathrm{H}$ NMR $\left(\mathrm{CDCl}_{3}, 300 \mathrm{MHz}\right): \delta 6.76-$ $6.63\left(\mathrm{~m}, 5 \mathrm{H}, \mathrm{C}_{6} \mathrm{H}_{5}\right), 5.04$ (br, s, $1 \mathrm{H}, \mathrm{OH}$, exchanged in

Table 2. Physicochemical data of synthesized 1,3 amino alcohols (2c-4c).

\begin{tabular}{llllll}
\hline Entry & Isoxazolidine & Reagent $^{\mathrm{a}}$ & Time $(\mathrm{min})$ & 1,3 amino alcohol $(\mathbf{2 c}-\mathbf{4 c})$ & Yield $(\%)$ \\
\hline 1 & 2a & Zn and dil acetic acid & 9 & 2c: Yellowish white thick liquid & 84 \\
2 & 3a & Zn and dil acetic acid & 10 & 3c: White thick liquid & 81 \\
3 & 4a & Zn and dil acetic acid & 10 & 4c: Dark gray thick liquid & 80
\end{tabular}

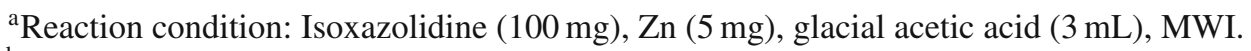

${ }^{\mathrm{b}}$ Isolated yields after purification 
$\left.\mathrm{D}_{2} \mathrm{O}\right), 4.80\left(\mathrm{~d}, 1 \mathrm{H}, J=3.76 \mathrm{~Hz}, \mathrm{C}_{5} \mathrm{H}\right), 4.15(\mathrm{dd}, 1 \mathrm{H}$, $\left.J=2.18,2.64 \mathrm{~Hz}, \mathrm{C}_{4} \mathrm{H}\right), 3.34$ (s, $3 \mathrm{H}, \mathrm{CH}_{3}$ protons), $2.70\left(\mathrm{dt}, 1 \mathrm{H}, J=3.10,2.80 \mathrm{~Hz}, \mathrm{C}_{3} \mathrm{H}\right), 2.08(\mathrm{dt} \sim \mathrm{m}$, $2 \mathrm{H}, \mathrm{CH}_{2}$ protons of $\left.-\mathrm{CH}_{2}-\left(\mathrm{CH}_{2}\right)_{3} \mathrm{OH}\right), 1.76-1.30(\mathrm{~m}$, $6 \mathrm{H}, \mathrm{CH}_{2}$ protons $) ;{ }^{13} \mathrm{C} \mathrm{NMR}\left(\mathrm{CDCl}_{3}\right): \delta 170.90,170.17$ (carbonyl carbons), 129.12, 128.75, 128.53, 127.33 (aromatic carbons), $85.56\left(\mathrm{C}_{5}\right), 73.21\left(\mathrm{C}_{3}\right), 63.08$ $\left(\mathrm{CH}_{2} \mathrm{OH}\right), 54.22\left(\mathrm{C}_{4}\right), 34.44\left(\mathrm{CH}_{3}\right), 23.63,22.70$, $22.12\left(3 \mathrm{CH}_{2}\right.$ carbons); FAB-MS: $m / z 304\left(\mathrm{M}^{+}\right), 289$, 231, 230, 227, 212, 154 (B.P), 77, 73; Anal. Found: C, 62.98; $\mathrm{H}, 6.40 ; \mathrm{N}, 9.09 . \mathrm{C}_{16} \mathrm{H}_{20} \mathrm{O}_{4} \mathrm{~N}_{2}$ requires $\mathrm{C}, 63.13$; $\mathrm{H}, 6.61 ; \mathrm{N}, 9.21 \%$.

2.1e Dihydro-3-(4-hydroxybutyl)-2,5-diphenyl-2Hpyrrolo[3,4-d]isoxazole-4,6(5H,6a-H)-dione (3a): Pale yellow solid. Yield 68\%; $\mathbf{R}_{f}=0.56$ (hexane-ethyl acetate, 5:1); IR (KBr): $v_{\max } 3585-3453(\mathrm{br}), 2920(\mathrm{~m})$, $2835(\mathrm{~m}), 1780$ (s), 1684 (s), 1600 (s), 1480 (m), $1346(\mathrm{~m}), 770(\mathrm{~s}) \mathrm{cm}^{-1} ;{ }^{1} \mathrm{H} \mathrm{NMR}\left(\mathrm{CDCl}_{3}, 300 \mathrm{MHz}\right)$ : $\delta$ 7.49-7.26 (m, 2X5H, $\left.\mathrm{C}_{6} \mathrm{H}_{5}\right), 6.84(\mathrm{br}, \mathrm{s}, 1 \mathrm{H}, \mathrm{OH}$, exchanged in $\left.\mathrm{D}_{2} \mathrm{O}\right), 4.95\left(\mathrm{~d}, 1 \mathrm{H}, J=6.52 \mathrm{~Hz}, \mathrm{C}_{5} \mathrm{H}\right)$, $3.91\left(\mathrm{dt}, 1 \mathrm{H}, J=6.04,6.16 \mathrm{~Hz}, \mathrm{C}_{3} \mathrm{H}\right), 3.55(\mathrm{dd}, 1 \mathrm{H}$, $\left.J=6.18,6.22 \mathrm{~Hz}, \mathrm{C}_{4} \mathrm{H}\right), 1.90\left(\mathrm{dt} \sim \mathrm{m}, 2 \mathrm{H}, \mathrm{CH}_{2}\right.$ protons of $\left.-\mathrm{CH}_{2}-\left(\mathrm{CH}_{2}\right)_{3} \mathrm{OH}\right), 1.63-1.18\left(\mathrm{~m}, 6 \mathrm{H}, 3 \mathrm{CH}_{2}\right.$ protons); ${ }^{13} \mathrm{C}$ NMR $\left(\mathrm{CDCl}_{3}\right): \delta 172.12,171.86$ (carbonyl carbons), 133.77, 133.14, 132.22, 131.78, 129.80, 129.61, 128.15, 127.72 (aromatic carbons), $87.80\left(\mathrm{C}_{5}\right)$, $75.18\left(\mathrm{C}_{3}\right), 65.27\left(\mathrm{CH}_{2} \mathrm{OH}\right), 56.37\left(\mathrm{C}_{4}\right), 34.55,26.40$, $25.00\left(3 \mathrm{CH}_{2}\right.$ carbons); FAB-MS: $m / z 366\left(\mathrm{M}^{+}\right), 306$, 293, 289, 216 (B.P), 77, 73, 59; Anal. Found: C, 68.67; $\mathrm{H}, 5.83 ; \mathrm{N}, 7.52 . \mathrm{C}_{21} \mathrm{H}_{22} \mathrm{O}_{4} \mathrm{~N}_{2}$ requires $\mathrm{C}, 68.82 ; \mathrm{H}$, $6.04 ; \mathrm{N}, 7.65 \%$.

\section{1f Dihydro-3-(4-hydroxybutyl)-2,5-diphenyl-2H-} pyrrolo[3,4-d]isoxazole-4,6(5H,6a-H)-dione

$(3 b)$ : White solid. Yield 25\%; $\mathbf{R}_{f}=0.48$ (hexane-ethyl acetate, 5:1); IR (KBr): $v_{\max }$ 3590-3476 (br), 2934 (m), $2830(\mathrm{~m}), 1780$ (s), 1680 (s), 1610 (s), 1476 (m), $1340(\mathrm{~m}), 776(\mathrm{~s}) \mathrm{cm}^{-1} ;{ }^{1} \mathrm{H} \mathrm{NMR}\left(\mathrm{CDCl}_{3}, 300 \mathrm{MHz}\right)$ : $\delta$ 7.36-7.15 (m, 2X5H, $\left.\mathrm{C}_{6} \mathrm{H}_{5}\right), 6.25(\mathrm{br}, \mathrm{s}, 1 \mathrm{H}, \mathrm{OH}$, exchanged in $\left.\mathrm{D}_{2} \mathrm{O}\right), 4.48\left(\mathrm{~d}, 1 \mathrm{H}, J=2.70 \mathrm{~Hz}, \mathrm{C}_{5} \mathrm{H}\right)$, $3.54\left(\mathrm{dt}, 1 \mathrm{H}, J=3.04,2.86 \mathrm{~Hz}, \mathrm{C}_{3} \mathrm{H}\right), 3.12(\mathrm{dd}, 1 \mathrm{H}$, $\left.J=2.26,2.18 \mathrm{~Hz}, \mathrm{C}_{4} \mathrm{H}\right), 1.84\left(\mathrm{dt} \sim \mathrm{m}, 2 \mathrm{H}, \mathrm{CH}_{2}\right.$ protons of $\left.-\mathrm{CH}_{2}-\left(\mathrm{CH}_{2}\right)_{3} \mathrm{OH}\right), 1.50-1.16\left(\mathrm{~m}, 6 \mathrm{H}, 3 \mathrm{CH}_{2}\right.$ protons); ${ }^{13} \mathrm{C} \mathrm{NMR}\left(\mathrm{CDCl}_{3}\right): \delta 170.63,170.14$ (carbonyl carbons), 131.62, 131.08, 130.27, 130.14, 129.19, 128.40, 128.05, 127.58 (aromatic carbons), $88.06\left(\mathrm{C}_{5}\right)$, $73.90\left(\mathrm{C}_{3}\right), 62.83\left(\mathrm{CH}_{2} \mathrm{OH}\right), 53.45\left(\mathrm{C}_{4}\right), 31.72,24.44$, 23.19 ( $3 \mathrm{CH}_{2}$ carbons); FAB-MS: $m / z 366\left(\mathrm{M}^{+}\right), 306$, 293, 289, 216 (B.P), 212, 77, 73, 59; Anal. Found: C, 68.63; H, 5.87; N, 7.60. $\mathrm{C}_{21} \mathrm{H}_{22} \mathrm{O}_{4} \mathrm{~N}_{2}$ requires $\mathrm{C}$, 68.82; $\mathrm{H}, 6.04 ; \mathrm{N}, 7.65 \%$. 2.1g 5-cycohexyl-dihydro-3-(4-hydroxybutyl)-2-phenyl2H-pyrrolo[3,4-d]isoxazole-4,6(5H,6 a-H)-dione (4a): Yellow crystal. Yield $62 \%, \mathbf{R}_{f}=0.50$ (hexane-ethyl acetate, 5:1); IR (KBr): $v_{\max }$ 3638-3515 (br), 2865 (s), 1785 (s), 1680 (s), 1446 (m), 1380 (m), 1265 (m), 780 (s) $\mathrm{cm}^{-1} ;{ }^{1} \mathrm{H}$ NMR $\left(\mathrm{CDCl}_{3}, 300 \mathrm{MHz}\right): \delta 7.40-7.17(\mathrm{~m}$, $\left.5 \mathrm{H}, \mathrm{C}_{6} \mathrm{H}_{5}\right), 5.94\left(\mathrm{~d}, 1 \mathrm{H}, J=6.64 \mathrm{~Hz}, \mathrm{C}_{5} \mathrm{H}\right), 4.40(\mathrm{br}, \mathrm{s}$, $1 \mathrm{H}, \mathrm{OH}$, exchanged in $\left.\mathrm{D}_{2} \mathrm{O}\right), 3.44(\mathrm{dd}, 1 \mathrm{H}, J=6.26$, $\left.6.08 \mathrm{~Hz}, \mathrm{C}_{4} \mathrm{H}\right), 2.23\left(\mathrm{dt}, 1 \mathrm{H}, J=6.60,6.14 \mathrm{~Hz}, \mathrm{C}_{3} \mathrm{H}\right)$, $1.69\left(\mathrm{dt} \sim \mathrm{m}, 2 \mathrm{H}, \mathrm{CH}_{2}\right.$ protons of $\left.-\mathrm{CH}_{2}-\left(\mathrm{CH}_{2}\right)_{3} \mathrm{OH}\right)$, $1.45-1.17\left(\mathrm{~m}, 17 \mathrm{H}\right.$, cyclohexyl and $\mathrm{CH}_{2}$ protons); ${ }^{13} \mathrm{C}$ NMR $\left(\mathrm{CDCl}_{3}\right): \delta 173.16,171.37$ (carbonyl carbons), 136.04, 135.52, 134.07, 133.93 (aromatic carbons), $86.80\left(\mathrm{C}_{5}\right), 77.08\left(\mathrm{C}_{3}\right), 62.50\left(\mathrm{CH}_{2} \mathrm{OH}\right), 55.62\left(\mathrm{C}_{4}\right)$, 38.51, 36.07, 34.40, 31.10, 29.52, 27.70, 26.30, 25.00, 23.28 (cyclohexyl and $\mathrm{CH}_{2}$ carbons); FAB-MS: $\mathrm{m} / \mathrm{z}$ $372\left(\mathrm{M}^{+}\right)$, 313, 299, 222 (B.P), 216, 83, 77, 73, 59; Anal. Found: C, 67.62; H, 7.43; N, 7.35. $\mathrm{C}_{21} \mathrm{H}_{28} \mathrm{O}_{4} \mathrm{~N}_{2}$ requires $\mathrm{C}, 67.71 ; \mathrm{H}, 7.56 ; \mathrm{N}, 7.52 \%$.

2.1h 5-cyclohexyl-dihydro-3-(4-hydroxybutyl)-2-phenyl2H-pyrrolo[3,4-d]isoxazole-4,6(5H,6 a-H)-dione (4b): Yellow crystal. Yield $31 \%, \mathrm{R}_{f}=0.44$ (hexane-ethyl acetate, 5:1); IR (KBr): $v_{\max }$ 3623-3534 (br), 2880 (s), 1782 (s), 1680 (s), 1440 (m), 1385 (m), 1260 (m), 774 (s) $\mathrm{cm}^{-1} ;{ }^{1} \mathrm{H} \mathrm{NMR}\left(\mathrm{CDCl}_{3}, 300 \mathrm{MHz}\right): \delta 7.26-7.08(\mathrm{~m}$, $\left.5 \mathrm{H}, \mathrm{C}_{6} \mathrm{H}_{5}\right), 4.90\left(\mathrm{~d}, 1 \mathrm{H}, J=2.66 \mathrm{~Hz}, \mathrm{C}_{5} \mathrm{H}\right), 4.56(\mathrm{br}, \mathrm{s}$, $1 \mathrm{H}, \mathrm{OH}$, exchanged in $\left.\mathrm{D}_{2} \mathrm{O}\right), 3.54(\mathrm{dd}, 1 \mathrm{H}, J=2.22$, $\left.2.50 \mathrm{~Hz}, \mathrm{C}_{4} \mathrm{H}\right), 2.40\left(\mathrm{dt}, 1 \mathrm{H}, J=3.08,3.04 \mathrm{~Hz}, \mathrm{C}_{3} \mathrm{H}\right)$, $1.60\left(\mathrm{dt} \sim \mathrm{m}, 2 \mathrm{H}, \mathrm{CH}_{2}\right.$ protons of $\left.-\mathrm{CH}_{2}-\left(\mathrm{CH}_{2}\right)_{3} \mathrm{OH}\right)$, 1.52-1.20 (m, $17 \mathrm{H}$, cyclohexyl and $\mathrm{CH}_{2}$ protons); ${ }^{13} \mathrm{C}$ NMR $\left(\mathrm{CDCl}_{3}\right): \delta 170.22,170.04$ (carbonyl carbons), 132.68, 132.30, 131.54, 131.38 (aromatic carbons), $85.10\left(\mathrm{C}_{5}\right), 76.20\left(\mathrm{C}_{3}\right), 63.44\left(\mathrm{CH}_{2} \mathrm{OH}\right), 56.70\left(\mathrm{C}_{4}\right)$, $37.32,36.40,33.69,31.28,30.55,28.12,26.90,25.43$, 24.20 (cyclohexyl and $\mathrm{CH}_{2}$ carbons); FAB-MS: $\mathrm{m} / \mathrm{z}$ $372\left(\mathrm{M}^{+}\right), 313,299,289,222$ (B.P), 216, 83, 77, 73, 59; Anal. Found: C, 67.57; H, 7.45; N, 7.30. $\mathrm{C}_{21} \mathrm{H}_{28} \mathrm{O}_{4} \mathrm{~N}_{2}$ requires $\mathrm{C}, 67.71 ; \mathrm{H}, 7.56 ; \mathrm{N}, 7.52 \%$.

2.1i General procedure for cycloaddition for regioisomers $(M W I)$ : A mixture of $N$-phenylhydroxylamine (250 mg, $2.29 \mathrm{mmol}$ ), 2,3-dihydro-4H-pyran (192 mg, 1 equivalent) was taken in a $25 \mathrm{~mL}$ Erlenmeyer flask, mixed well and subjected to microwave irradiation at $250 \mathrm{~W}$ for $5 \mathrm{~min}$. The formation of nitrone was monitored by TLC (hexane:ethyl acetate $=5: 1 ; \mathrm{R}_{f}=0.38$ ). Ethyl acrylate ( $229 \mathrm{mg}, 1$ equivalent) was added at this stage and the reaction mixture was further irradiated at $250 \mathrm{~W}$ for appropriate time (table 1). After completion of the reaction, as indicated by TLC (hexane:ethyl acetate $=5: 1 ; \mathbf{R}_{f}=0.52$ ), the reaction mixture was 
cooled to RT and washed with diethyl ether $(3 \times$ $10 \mathrm{~mL}$ ). The combined ether extract was concentrated in vacuo and the resulting product was directly charged on silica gel column and eluted with a mixture of ethyl acetate:hexane to afford pure isoxazolidine derivative $\mathbf{5}$ (entry 4, table 1). This procedure was followed for other substrate listed in table 1 .

\subsection{Ethyl 3-(4-hydroxy butyl)-2-phenyl isoxazolidine-5-carboxylate (5)}

Colourless thick liquid. Yield 92\%, $\mathrm{R}_{f}=0.52$ (hexaneethyl acetate, 5:1); IR (KBr): $v_{\max }$ 3610-3525 (br), 2930 (s), 2842 (m), 1760 (s), 1444 (s), 790 (s) cm ${ }^{-1}$; ${ }^{1} \mathrm{HNMR}\left(\mathrm{CDCl}_{3}, 300 \mathrm{MHz}\right): \delta \quad 7.24-7.08(\mathrm{~m}, 5 \mathrm{H}$, $\mathrm{C}_{6} \mathrm{H}_{5}$ ), 4.92-4.80 (br, s, $1 \mathrm{H},-\mathrm{OH}$, exchanged in $\mathrm{D}_{2} \mathrm{O}$ ), $4.20\left(\mathrm{q}, 2 \mathrm{H}, J=6.10,6.06 \mathrm{~Hz},-\mathrm{OCH}_{2} \mathrm{CH}_{3}\right), 4.06$ (t, $\left.1 \mathrm{H}, J=6.68 \mathrm{~Hz}, \mathrm{C}_{5} \mathrm{H}\right), 3.40(\mathrm{ddd}, 1 \mathrm{H}, J=6.54$, $\left.6.58 \mathrm{~Hz}, \mathrm{C}_{3} \mathrm{H}\right), 3.18(\mathrm{dd}, 2 \mathrm{H}, J=6.12,6.14 \mathrm{~Hz}$, $\left.\mathrm{C}_{4} 2 \mathrm{H}\right), 1.68\left(\mathrm{dt} \sim \mathrm{m}, 2 \mathrm{H}, \mathrm{CH}_{2}\right.$ protons of $-\underline{\mathrm{CH}}_{2}-$ $\left.\left(\mathrm{CH}_{2}\right)_{3} \mathrm{OH}\right), 1.26\left(\mathrm{t}, 3 \mathrm{H}, J=5.40 \mathrm{~Hz},-\mathrm{OCH}_{2} \mathrm{CH}_{3}\right)$, 1.18-0.82 (m, $6 \mathrm{H}, \mathrm{CH}_{2}$ protons); ${ }^{13} \mathrm{C}$ NMR $\left(\mathrm{CDCl}_{3}\right)$ : $\delta 169.77$ (carbonyl carbon), 130.72, 130.54, 130.27, 129.88 (aromatic carbons), $84.16\left(\mathrm{C}_{5}\right), 79.62\left(\mathrm{C}_{3}\right)$, $66.40\left(\mathrm{CH}_{2} \mathrm{OH}\right), 61.52\left(\mathrm{CH}_{2}\right.$ carbon of $\left.-\mathrm{OCH}_{2} \mathrm{CH}_{3}\right)$, $56.86\left(\mathrm{C}_{4}\right), 22.28,21.68,20.94\left(3 \mathrm{CH}_{2}\right.$ carbons $), 16.40$ $\left(\mathrm{CH}_{3}\right.$ carbon of $\left.\mathrm{OCH}_{2} \underline{\mathrm{CH}}_{3}\right)$; FAB-MS: $m / z 293\left(\mathrm{M}^{+}\right)$, 220, 219, 143 (B.P), 111, 77, 73; Anal. Found: C, 65.22; $\mathrm{H}, 7.48 ; \mathrm{N}, 4.54 . \mathrm{C}_{16} \mathrm{H}_{23} \mathrm{O}_{4} \mathrm{~N}$ requires $\mathrm{C}, 65.49 ; \mathrm{H}, 7.89$; $\mathrm{N}, 4.77 \%$.

2.2a 4-(2,5-diphenyl-isoxazolidin-3-yl)butan-1-ol (6): Colourless thick liquid. Yield 91\%, $\mathrm{R}_{f}=0.50$ (hexaneethyl acetate, 5:1); IR (KBr): $v_{\max } 3620-3565$ (br), 2925 (s), 2844 (m), 1710 (s), 1440 (m), 1324 (s), $804(\mathrm{~m}), 776(\mathrm{~s}) \mathrm{cm}^{-1} ;{ }^{1} \mathrm{H} \mathrm{NMR}\left(\mathrm{CDCl}_{3}, 300 \mathrm{MHz}\right)$ : $\delta$ 6.93-6.56 (m, 2X5H, $\mathrm{C}_{6} \mathrm{H}_{5}$ protons), $5.02(\mathrm{t}, 1 \mathrm{H}$, $\left.J=6.08 \mathrm{~Hz}, \mathrm{C}_{5} \mathrm{H}\right), 4.85-4.76(\mathrm{br}, \mathrm{s}, 1 \mathrm{H}$, exchanged in $\mathrm{D}_{2} \mathrm{O}$ ), $4.28\left(\mathrm{ddd}, 1 \mathrm{H}, J=6.52,6.50 \mathrm{~Hz}, \mathrm{C}_{3} \mathrm{H}\right), 3.87$ $\left(\mathrm{dd}, 2 \mathrm{H}, J=6.22,6.20 \mathrm{~Hz}, \mathrm{C}_{4} 2 \mathrm{H}\right), 1.96(\mathrm{dt} \sim \mathrm{m}, 2 \mathrm{H}$, $\mathrm{CH}_{2}$ protons of $\left.-\mathrm{CH}_{2}-\left(\mathrm{CH}_{2}\right)_{3} \mathrm{OH}\right), 1.50-1.24(\mathrm{~m}, 6 \mathrm{H}$, $\mathrm{CH}_{2}$ protons); ${ }^{13} \mathrm{C} \mathrm{NMR}\left(\mathrm{CDCl}_{3}\right): \delta 136.75,136.52$, $136.17,135.94,131.88,131.54,131.12,130.82$ (aromatic carbons), $85.95\left(\mathrm{C}_{5}\right), 77.32\left(\mathrm{C}_{3}\right), 60.64\left(\mathrm{CH}_{2} \mathrm{OH}\right)$, $56.40\left(\mathrm{C}_{4}\right), 21.48,20.10,19.63\left(3 \mathrm{CH}_{2}\right.$ carbons $)$; FABMS: $m / z 297\left(\mathrm{M}^{+}\right), 265,224,219,147$ (B.P), 77, 73; Anal. Found: C, 76.60; H, 7.47; N, 4.63. $\mathrm{C}_{19} \mathrm{H}_{23} \mathrm{O}_{2} \mathrm{~N}$ requires $\mathrm{C}, 76.73 ; \mathrm{H}, 7.78 ; \mathrm{N}, 4.71 \%$.

2.2b General procedure for synthesis of 1,3 amino alcohols (MWI): A mixture of isoxazolidine $\mathbf{2 a}$ $(100 \mathrm{mg})$ and $\mathrm{Zn}$ dust $(5 \mathrm{mg})$ in dil acetic acid $(3 \mathrm{~mL})$ was taken in a $25 \mathrm{~mL}$ Erlenmeyer flask, mixed well and subjected to microwave irradiation at $250 \mathrm{~W}$ for appropriate time (entry 1 , table 2 ). The completion of reaction was monitored by TLC ((hexane:ethyl acetate, 5:1; $\mathbf{R}_{f}=0.66$ ). The reaction mixture was cooled to RT, extracted with diethyl ether and filtered. Excess acetic acid in the filtrate was removed through basic work up and finally charged on silica gel column and eluted with a mixture of ethyl acetate:hexane to afford pure 1,3 amino alcohol in $84 \%$ yield (entry 1 ; scheme 2; table 2). This procedure was followed for other substrates listed in table 2.

\section{2c 3-hydroxy-4-(5-hydroxy-1-(phenylamino)pentyl)-}

1-methylpyrrolidine-2,5-dione (2c): Yellowish white thick liquid. Yield $84 \%, \mathrm{R}_{f}=0.66$ (hexane-ethyl acetate, 5:1); IR (KBr): $v_{\max }$ 3650-3585 (br), 3510 3435 (br), 2845 (m), 1782 (s), 1685 (m), 1490 (m), $783(\mathrm{~s}) \mathrm{cm}^{-1} ;{ }^{1} \mathrm{H}$ NMR $\left(\mathrm{CDCl}_{3}, 300 \mathrm{MHz}\right): \delta 7.05$ $6.88\left(\mathrm{~m}, 5 \mathrm{H}, \mathrm{C}_{6} \mathrm{H}_{5}\right), 5.24(\mathrm{br}, 2 \mathrm{H}, 2 \mathrm{XOH}$, exchanged in $\left.\mathrm{D}_{2} \mathrm{O}\right), 4.52\left(\mathrm{~d}, 1 \mathrm{H}, J=6.50 \mathrm{~Hz}, \mathrm{C}_{1} \mathrm{H}\right), 3.80(\mathrm{dd}, 1 \mathrm{H}$, $\left.J=6.54,6.30 \mathrm{~Hz}, \mathrm{C}_{2} \mathrm{H}\right), 3.50\left(\mathrm{br}, 1 \mathrm{H},-\mathrm{NHC}_{6} \mathrm{H}_{5}\right)$, $3.36\left(\mathrm{~s}, 3 \mathrm{H}, \mathrm{CH}_{3}\right.$ proton), $2.90(\mathrm{dt}, 1 \mathrm{H}, J=6.36$, $\left.6.28 \mathrm{~Hz}, \mathrm{C}_{3} \mathrm{H}\right), 2.64\left(\mathrm{dt} \sim \mathrm{m}, 2 \mathrm{H}, \mathrm{CH}_{2}\right.$ protons of $\left.\mathrm{CH}_{2}-\left(\mathrm{CH}_{2}\right)_{3} \mathrm{OH}\right), 2.12-1.65$ (m, $6 \mathrm{H}, \mathrm{CH}_{2}$ protons); ${ }^{13} \mathrm{C}$ $\mathrm{NMR}\left(\mathrm{CDCl}_{3}\right): \delta 173.70,172.66$ (carbonyl carbons), 129.94, 129.66, 129.14, 128.85 (aromatic carbons), $86.54\left(\mathrm{C}_{1}\right), 74.62\left(\mathrm{C}_{3}\right), 66.30\left(\mathrm{CH}_{2} \mathrm{OH}\right), 55.08\left(\mathrm{C}_{2}\right)$, $33.12\left(\mathrm{CH}_{3}\right), 26.50,24.22,22.77$ (3 $\mathrm{CH}_{2}$ carbons); FAB-MS: $m / z 306\left(\mathrm{M}^{+}\right), 233,229,156,77,73$; Anal. Found: $\mathrm{C}, 62.36 ; \mathrm{H}, 7.06 ; \mathrm{N}, 9.01 . \mathrm{C}_{16} \mathrm{H}_{22} \mathrm{O}_{4} \mathrm{~N}_{2}$ requires C, 62.74; H, 7.23; N, 9.15\%.

2.2d 3-hydroxy-4-(5-hydroxy-1-(phenylamino)pentyl)-1phenyl pyrrolidine-2,5-dione (3c): White thick liquid. Yield 81\%, $\mathbf{R}_{f}=0.68$ (hexane-ethyl acetate, 5:1); IR (KBr): $v_{\max } 3670-3590$ (br), 3505-3420 (br), 2840 (m), 1780 (s), 1680 (m), 1486 (m), 775 (s) cm ${ }^{-1} ;{ }^{1} \mathrm{H}$ NMR $\left(\mathrm{CDCl}_{3}, 300 \mathrm{MHz}\right): \delta 7.22-7.08\left(\mathrm{~m}, 2 \mathrm{X} 5 \mathrm{H}, \mathrm{C}_{6} \mathrm{H}_{5}\right)$, 5.18-5.00 (br, $2 \mathrm{H}, 2 \mathrm{XOH}$, exchanged in $\mathrm{D}_{2} \mathrm{O}$ ), 4.80 $\left(\mathrm{d}, 1 \mathrm{H}, J=6.74 \mathrm{~Hz}, \mathrm{C}_{1} \mathrm{H}\right), 3.76(\mathrm{dd}, 1 \mathrm{H}, J=6.30$, $\left.6.38 \mathrm{~Hz}, \mathrm{C}_{2} \mathrm{H}\right), 3.43\left(\mathrm{br}, 1 \mathrm{H},-\mathrm{NHC}_{6} \mathrm{H}_{5}\right), 2.78(\mathrm{dt}, 1 \mathrm{H}$, $\left.J=6.44,6.40 \mathrm{~Hz}, \mathrm{C}_{3} \mathrm{H}\right), 2.55\left(\mathrm{dt} \sim \mathrm{m}, 2 \mathrm{H}, \mathrm{CH}_{2}\right.$ protons of $\left.-\mathrm{CH}_{2}-\left(\mathrm{CH}_{2}\right)_{3} \mathrm{OH}\right), 1.95-1.40\left(\mathrm{~m}, 6 \mathrm{H}, \mathrm{CH}_{2}\right.$ protons $)$; ${ }^{13} \mathrm{C}$ NMR $\left(\mathrm{CDCl}_{3}\right): \delta$ 174.08, 172.55 (carbonyl carbons), 135.80, 135.47, 133.54, 133.11, 132.12, 130.80, 130.23, 129.90 (aromatic carbons), $85.66\left(\mathrm{C}_{1}\right), 75.20$ $\left(\mathrm{C}_{3}\right), 63.90\left(\mathrm{CH}_{2} \mathrm{OH}\right), 55.30\left(\mathrm{C}_{2}\right), 27.03,24.80,22.13$ (3 $\mathrm{CH}_{2}$ carbons); FAB-MS: $m / z 368\left(\mathrm{M}^{+}\right), 295,291$, 218, 214, 77, 73; Anal. Found: C, 68.33; H, 6.25; N, 7.28. $\mathrm{C}_{21} \mathrm{H}_{24} \mathrm{O}_{4} \mathrm{~N}_{2}$ requires $\mathrm{C}, 68.47 ; \mathrm{H}, 6.52 ; \mathrm{N}, 7.60 \%$. 
2.2e 1-cyclohexyl-3-hydroxy-4-(5-hydroxy-1-(phenylamino)pentyl)pyrrolidine-2,5-dione (4c): Dark gray thick liquid. Yield $80 \%, \mathrm{R}_{f}=0.64$ (hexane-ethyl acetate, 5:1); IR (KBr): $v_{\max }$ 3665-3580 (br), 35003425 (br), 2845 (m), 1778 (s), 1684 (m), 1440 (m), $1210(\mathrm{~m}), 770$ (s) $\mathrm{cm}^{-1} ;{ }^{1} \mathrm{H} \mathrm{NMR}\left(\mathrm{CDCl}_{3}, 300 \mathrm{MHz}\right)$ : $\delta$ 6.83-6.66 (m, 5H, $\left.\mathrm{C}_{6} \mathrm{H}_{5}\right), 5.22-5.09(\mathrm{br}, 2 \mathrm{H}, 2 \mathrm{XOH}$, exchanged in $\left.\mathrm{D}_{2} \mathrm{O}\right), 4.70\left(\mathrm{~d}, 1 \mathrm{H}, J=6.50 \mathrm{~Hz}, \mathrm{C}_{1} \mathrm{H}\right)$, $3.90\left(\mathrm{dd}, 1 \mathrm{H}, J=6.88,6.76 \mathrm{~Hz}, \mathrm{C}_{2} \mathrm{H}\right), 3.33(\mathrm{br}, 1 \mathrm{H}$, $\left.-\mathrm{NHC}_{6} \mathrm{H}_{5}\right), 2.60\left(\mathrm{dt}, 1 \mathrm{H}, J=6.70,6.26 \mathrm{~Hz}, \mathrm{C}_{3} \mathrm{H}\right)$, $2.28\left(\mathrm{dt} \sim \mathrm{m}, 2 \mathrm{H}, \mathrm{CH}_{2}\right.$ protons of $\left.-\mathrm{CH}_{2}-\left(\mathrm{CH}_{2}\right)_{3} \mathrm{OH}\right)$, 1.90-0.96 (m, $17 \mathrm{H}, \mathrm{CH}_{2}$ protons); ${ }^{13} \mathrm{C} \mathrm{NMR}\left(\mathrm{CDCl}_{3}\right)$ : $\delta$ 170.20, 170.05 (carbonyl carbons), 128.90, 128.56, 128.05, 127.66 (aromatic carbons), $86.22\left(\mathrm{C}_{1}\right), 74.28$ $\left(\mathrm{C}_{3}\right), 62.50\left(\mathrm{CH}_{2} \mathrm{OH}\right), 56.80\left(\mathrm{C}_{2}\right), 29.00,28.45,28.19$, 27.06, 25.60, 23.86, 22.32, 20.72, $20.16\left(\mathrm{CH}_{2}\right.$ carbons); FAB-MS: $m / z 374\left(\mathrm{M}^{+}\right), 301,291,224,214$, 83, 77, 73; Anal. Found: C, 67.18; H, 7.88; N, 7.26. $\mathrm{C}_{21} \mathrm{H}_{30} \mathrm{O}_{4} \mathrm{~N}_{2}$ requires $\mathrm{C}, 67.37 ; \mathrm{H}, 8.02 ; \mathrm{N}, 7.48 \%$.

\section{Results and discussion}

In the present study, the formation of nitrone $\mathbf{1}$ has been achieved by treating 2,3-dihydro- $4 \mathrm{H}$-pyran with $\mathrm{N}$ phenylhydroxylamine under microwave irradiation and has been trapped in situ by various activated alkenes in 1,3-dipolar cycloaddition reaction with moderate selectivity and high yield resulting new isoxazolidine derivatives (2-6) (scheme 1; table 1). Dimerization of nitrone could be controlled under this condition.

Induction of three asymmetric centres at $\mathrm{C}_{5}, \mathrm{C}_{4}$ and $\mathrm{C}_{3}$ positions of the newly developed isoxazolidine derivatives have made this one-pot synthesis highly attractive. The development of diastereomers can be rationalized by an exo approach of nitrone 1 which has $Z$ configuration for the formation of major cycloadducts $\mathbf{2 a - 4 a}$ (transition state 1). The minor cycloadducts $\mathbf{2 b}-\mathbf{4 b}$ are formed by the endo approach of $Z$ nitrone (transition state 2). The mixture of diastereomers are identified by considering the multiplicity of the proton signals at 3-H and 4-H along with their coupling constant values. ${ }^{30,31}$ The most significant differences in the ${ }^{1} \mathrm{H}$ NMR data for the diastereomers are the position and multiplicity of the 3-H signal. In the major adducts $\mathbf{2 a}-\mathbf{4 a}$, coupling constant between $3-\mathrm{H}$ and $4-\mathrm{H}$ has been measured as $J_{3,4} \sim 6.26 \mathrm{~Hz}$ whilst for minor adducts $\mathbf{2 b}-\mathbf{4} \mathbf{b}, J_{3,4}$ is $\sim 2.54 \mathrm{~Hz}$. These differences can be explained by considering the available isoxazolidine ring conformations. Due to the 4,5-fused pyrrolidindione, the isoxazolidine ring adopts an envelope conformation and allowing for inversion, its nitrogen atom will either extend out from the envelope, i.e., minor conformation, or point inside the envelope, i.e., major conformation. The minor conformer has the $N$ lone pair antiperiplanar and therefore, capable of shielding 3- $H$ proton, so this conformation is assigned to the minor conformer (figure 1). The diastereomeric isoxazolidines $\mathbf{2 a - 4 a}$ and $\mathbf{2 b}-\mathbf{4} \mathbf{b}$ were separated by column chromatography and obtained in analytically pure form by recrystalization from heptane-ethyl acetate.

In all the diastereomers, the configurations of H-5 and $\mathrm{H}-4$ are cis as evidenced from their coupling constant values. The cycloaddition reactions of $\mathrm{N}$-phenyl5-hydroxy nitrone with ethyl acrylate and styrene are found to be regioselective and results 5-substituted isoxazolidine derivatives exclusively. This was rationalized by using frontier molecular orbital theory and ${ }^{1} \mathrm{H}$ NMR experiments. The results remind us the pioneering works on the study of regioselectivity in cycloaddition reactions by Houk et al. and Huisgen which state that exclusively regioselective cycloadducts are possible only when LUMO (nitrone)-HOMO (dipolarophile) interactions completely dominate the reaction and lead to the formation of only 5-substituted adducts. ${ }^{32-34}$ From the ${ }^{1} \mathrm{H}$ NMR spectrum of cycloadducts 5-6, it has been found that clear double-doublet signal for $\mathrm{H}-4$ protons and double triplet (ddd) signal for $\mathrm{H}-3$ protons are obtained in all the cases due to further coupling from vicinal hydrogens and hence confirms in favour of 5substituted adducts. From the detailed investigations on the nature of these cycloaddition reactions using TLC and ${ }^{1} \mathrm{H}$ NMR spectrum studies for the cycloadducts $\mathbf{5}_{-}$ $\mathbf{6}$, it is also confirmed that no diastereomers are formed. The relative configurations of $\mathrm{H}-3, \mathrm{H}-4$ and $\mathrm{H}-5$ protons in these adducts are syn and the cycloadducts
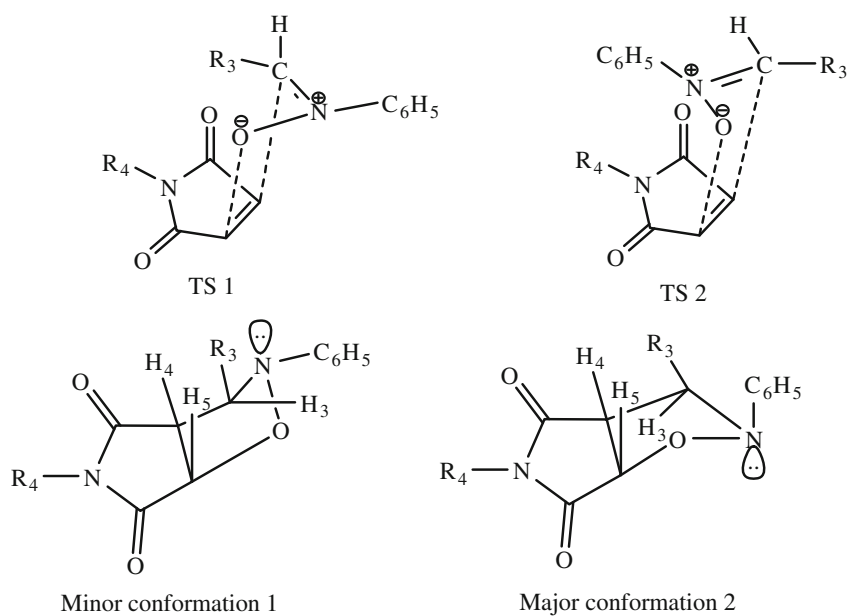

$\mathrm{R}_{3}=\left(\mathrm{CH}_{2}\right)_{4} \mathrm{OH} ; \mathrm{R}_{4}=\mathrm{Ph} ; \mathrm{Me} ; \mathrm{Cy}$

Figure 1. TS for the development of cycloadducts and their conformations. 
are in favour of exo transition state geometry as evidenced from their coupling constant values $\left(J_{\mathrm{H} 4, \mathrm{H} 5}=\right.$ $6.08-6.52 \mathrm{~Hz} ; J_{\mathrm{H} 4, \mathrm{H} 3}=6.10-6.68 \mathrm{~Hz}$ ).

The newly developed $N$-phenyl isoxazolidine derivatives $(\mathbf{2} \mathbf{a}-\mathbf{4 a})$ can be easily converted into acyclic chiral 1,3 difunctional amino alcohols (2c-4c) (scheme 2, table 2) by the reductive cleavage of the $\mathrm{N}-\mathrm{O}$ bond. These conversions have been achieved by simply treating the substrates with zinc powder in dil acetic acid under microwave irradiation. ${ }^{27-29}$

The relative configurations of $\mathrm{H}-1, \mathrm{H}-2$ and $\mathrm{H}-3$ protons of the newly developed 1,3 amino alcohols (2c4c) are syn as evidenced from their coupling constant values $\left(J_{\mathrm{H} 1, \mathrm{H} 2} \sim 6.70 \mathrm{~Hz} ; J_{\mathrm{H} 2, \mathrm{H} 3} \sim 6.10 \mathrm{~Hz}\right)$. Expected broad signals for $\mathrm{N}-\mathrm{H}$ proton around $\delta 3.40$ and alcoholic $\mathrm{OH}$ groups around $\delta 5.20 \mathrm{ppm}$ are also obtained. Synthesis of 1,3 amino alcohols using other isoxazolidine derivatives $(\mathbf{2 b}-\mathbf{4 b} ; \mathbf{5 , 6})$ are in progress. In general, all the reactions are very clean and high yielding compared to conventional cycloaddition reactions of nitrones. ${ }^{24-26}$ The products have been characterized from their spectroscopic (IR, ${ }^{1} \mathrm{H} \mathrm{NMR},{ }^{13} \mathrm{C} \mathrm{NMR}, \mathrm{MS}$ ) data. No catalyst or co-organic solvent is required.

\section{Conclusion}

This paper represents an extension of our studies dealing with the synthesis of heterocyclic compounds having isoxazolidine and isoxazoline cores in their structure. Further studies will be carried out in the near future to test their potential biological activities.

\section{Acknowledgements}

We thank Dr. M P Kharel, Principal, Sikkim Government College for providing facilities and constant encouragement. We acknowledge the financial support from the University Grants Commission (UGC), New Delhi (grant no:34-304/2008-SR) and also to the Central Drug Research Institute (CDRI), Lucknow for providing spectral data.

\section{References}

1. Lidström P, Tierney J, Wathey B and Westman J 2001 Tetrahedron $\mathbf{5 7} 9225$

2. Kappe C O 2004 Angew. Chem. Int. Ed. 436250

3. Perreux L and Loupy A 2001 Tetrahedron 579199

4. Loupy A, Petti A, Hamelin J, Texier-Boullet F, Jacquault $\mathrm{P}$ and Mathé D 1998 Synthesis 91213
5. Loupy A 2006 Microwaves in organic synthesis (Weinheim, Germany: Wiley-VCH) 2nd edn

6. Kabalka G W and Pagni R M 1997 Tetrahedron 537999

7. Varma R S 1999 Green Chem. 143

8. Pineiro M and Teresa M V D Pinho e Melo 2009 Eur. J. Org. Chem. 5287

9. De La Hoz A, Diaz-Ortis A, Moreno A and Langa F 2000 Eur. J. Org. Chem. 3659

10. Meng L, Fettinger J C and Kurth M J 2007 Org. Lett. 9 5055

11. Chiacchio U, Corsaro A, Iannazzo D, Piperno A, Romeo G, Romeo R, Saita M G and Rescifina A 2007 Eur. J. Org. Chem. 4758

12. Mabrour M, Bougrin K, Benhida R, Loupy A and Saufiaoui M 2007 Tetrahedron Lett. 48443

13. Andrade M M and Barros M T 2009 Arkivoc. 11299

14. Frederickson M 1997 Tetrahedron $\mathbf{5 3} 403$

15. Chakraborty B, Sharma P K, Rai N, Kafley S and Chhetri M S 2010 J. Chem. Res. 34(3) 147

16. Chakraborty B, Kafley S, Chhetri M S and Samanta A 2010 Indian J. Chem. 49B 209

17. Chakraborty B and Chhetri M S 2010 Indian J. Chem. 49B 102

18. Chakraborty B, Chhetri M S and Samanta A 2010 Indian J. Chem. 49B 1155

19. Chakraborty B and Sharma P K 2011 Synth. Commmun. (MS No: LSYC-2010-544833, in production house for online publication)

20. Chakraborty B, Sharma P K and Chhetri M S 2011 J. Heterocycl. Chem. (Wiley) DOI:10.1002/jhet.971, releasing online soon

21. Chakraborty B and Chhetri M S 2008 Indian J. Chem. 47B 485

22. Chakraborty B, Sharma P K, Kafley S, Chhetri M S and Ghosh A R 2011 J. Indian Chem. Soc. 88245

23. Chakraborty B, Sharma P K, Chhetri M S, Kafley S and Rai N 2010 J. Indian Chem. Soc. 871399

24. Pandey G, Banerjee P and Gadre S R 2006 Chem. Rev. 1064484

25. Molteni G 2006 Heterocycles 682177

26. Padwa A and Pearson W H 2003 Synthetic applications of 1,3-Dipolar cycloaddition chemistry towards heterocycles and natural products (Hoboken NJ: John Wiley and Sons)

27. Hoffman W R, Eichler G and Endesfelder A 1983 Liebigs Ann. Chem. 2000

28. Yepez A F, Palma A, Stashenko E, Bahsas A and AmaroLuis J M 2006 Tetrahedron Lett. 47(32) 5825

29. Marini F, Tiecco M, Testaferri L, Sternatiovo S, Santi C, Bagnoli L and Temperini A 2001 Tetrahedron Asymm. 123053

30. Deshong P, Li W, Kennington J W Jr, Ammon H L and Leginus J M 1991 J. Org. Chem. 561364

31. Yu Y, Ohno M and Eguchi S 1993 Tetrahedron 49 823

32. Houk K N and Moses S R 1984 J. Am. Chem. Soc. 106 3880

33. Huisgen R 1976 J. Org. Chem. 41403

34. Houk K N, Sims J and Luskus C R 1973 J. Am. Chem. Soc. 957302 